\title{
Modificación de las propiedades de matrices cementantes mediante la adición de partículas de nanosílice
}

\author{
L. Y. Gómez-Zamorano ${ }^{1}$, C. E. Castillo-Linton ${ }^{1}$
}

${ }^{1}$ Universidad Autónoma de Nuevo León, Facultad de Ingeniería Mecánica y Eléctrica, Programa Doctoral en Ingeniería de Materiales, Ave. Universidad s/n, Ciudad Universitaria, San Nicolás de los Garza, Nuevo León, México, CP 66450.

\begin{tabular}{l} 
Información del artículo \\
DOI: \\
http://dx.doi.org/10.21041/ra.v \\
\hline 6i2.132 \\
Artículo recibido el 08 de \\
febrero de 2016, revisado bajo \\
las políticas de publicación de \\
la Revista ALCONPAT y \\
aceptado el 10 de abril de \\
2016. Cualquier discusión, \\
incluyendo la réplica de los \\
autores, se publicará en el \\
primer número del año 2017 \\
siempre y cuando la \\
información se reciba antes del \\
cierre del tercer número del \\
año 2016.
\end{tabular}

(c) 2016 ALCONPAT Internacional

Información Legal

Revista ALCONPAT, Año 6, No. 2 ,

Mayo - Agosto 2016, es una publicación cuatrimestral de la Asociación

cuatrimestral de la Asociación

Patología y Recuperación de la

Construcción, Internacional, A.C., Km. 6 ,

antigua carretera a Progreso, Mérida

Yucatán, C.P. 97310, Tel.5219997385893

, alconpat.int@gmail.com, Página Web: www.revistaalconpat.org

Editor responsable: Dr. Pedro Castro

Borges. Reserva de derechos al uso

exclusivo No.04-2013-011717330300-

exclusivo No.04-2013-011717330300-

por el Instituto Nacional de Derecho de

Autor. Responsable de la última

actualización de este número, Unidad de

Informática ALCONPAT, Ing. Elizabeth

Sabido Maldonado, Km. 6, antigua

carretera a Progreso, Mérida Yucatán,

C.P. 97310 , fecha de última modificación:

30 de mayo de 2016.

Las opiniones expresadas por los autores no necesariamente reflejan la postura del editor.

Queda totalmente prohibida la

reproducción total o parcial de los

contenidos e imágenes de la publicación

sin previa autorización de la ALCONPAT

Internacional A.C

\section{RESUMEN}

Este trabajo de investigación evaluó el efecto de la adición de nanopartículas de sílice (NS) a dos matrices cementantes, base cemento portland ordinario y cemento sulfoaluminoso, con el fin de establecer su influencia en las propiedades mecánicas y de resistencia química de dichos materiales. Para esto, se adicionaron las NS en dosificaciones de $0.30 \%$ a $5.0 \%$ en peso. Los resultados indicaron que la resistencia a la compresión y al ataque químico por sulfatos, se ven mejoradas debido a la adición de NS. La resistencia al ataque químico por sulfatos se mejoró de forma importante con la presencia de NS en comparación al cemento sin adiciones. Este resultado sugiere que ambas matrices presentaron una mayor densificación.

Palabras claves: Reactividad, materiales cementosos suplementarios.

\section{ABSTRACT}

This research focused on evaluating the effect of adding silica nanoparticles (NS) to two cementitious matrices, as ordinary portland and sulfoaluminate cement, in order to establish their influence on the mechanical and chemical properties. To conduct this evaluation, the NS were added in dosages of 0.30to-5.0\% by mass relative to cement. The results indicated that the compressive strength and resistance to chemical attack by sulfates were improved due to the addition of silica nanoparticles, in both matrices. Finally, the resistance to chemical attack by sulfates showed an improvement with the addition of silica nanoparticles when comparing with pure cement, suggesting an increase in the densification.

Keywords: Reactivity, supplementary cementitious materials.

\section{RESUMO}

Este estudo avaliou o efeito da adição de nanopartículas de sílica (NS) em duas matrizes cimentícias, base cimento Portland comum e cimento sulfoaluminoso, a fim de estabelecer a sua influência sobre as propriedades de resistência mecânica e química dos referidos materiais. Para isso, foram adicionadas as NS em dosagens de $0,30 \%$ a 5,0\% em massa. Os resultados indicaram que a resistência à compressão e ao ataque químico por sulfatos são aumentadas devido à adição do NS. A resistência ao ataque químico por sulfatos melhorou significativamente com a presença de NS em comparação com o cimento sem adições. Este resultado sugere que ambas as matrizes apresentaram uma maior densificação.

Palavras-clave: Reatividade, materiais cimentícios suplementares.

Autor de contacto: Lauren Y. Gómez Zamorano (lauren.gomezzm@uanl.edu.mx) 
Revista ALCONPAT, Volumen 6, Número 2, Mayo - Agosto 2016, Páginas 101 - 115

\section{INTRODUCCIÓN}

Entre las investigaciones recientes en la industria de la construcción, es posible identificar el uso de nanomateriales que, adicionados a las matrices cementantes, tienen como objetivo modificar las propiedades reológicas de los concretos. Algunos de los compuestos más comúnmente usados son: nanosílices, nanocompuestos fotocatalíticos como el $\mathrm{TiO}_{2}$, nano $\mathrm{CaCO}_{3}$ y nanoarcillas; además se han investigado para el desarrollo de materiales híbridos, donde las nanopartículas no son adicionadas al cemento, sino que se forman en la matriz cementante.

Otros tipos de materiales que han sido probados, son nanofibras de carbono, nano fibras de celulosa y se ha trabajado también con el uso de nanomateriales con la finalidad de modificar las propiedades de los agregados utilizados para la fabricación de cemento. Uno de los materiales más utilizados a nivel nanométrico es la sílice (nanopartículas de sílice, NS), debido a su comportamiento puzolánico. Los resultados en general concuerdan que al utilizar este material, las propiedades mecánicas se ven mejoradas, tal como lo reportó (Sobolev et. al., 2009), quienes adicionaron NS de tamaños de 5 a 70 $\mathrm{nm}$ encontrando que tanto la resistencia a la compresión (RC) como la resistencia a la flexión de los morteros fabricados con una adición de $0.25 \%$ en peso de partículas de nano- $\mathrm{SiO}_{2}$ con respecto al cemento portland, se vieron mejoradas en porcentajes de $16 \%$ a edades de $24 \mathrm{~h}$ y de $18 \%$ a $28 \mathrm{~h}$ respectivamente. Otros estudios (Belkowitz et. al., 2010), se han enfocado en la comparación de las propiedades obtenidas en el concreto al utilizar partículas de microsilica y compararlas con NS (Mondal et. al. 2010, Shah et.al., 2009), en donde encontraron que al añadir NS en concreto en porcentajes de entre $0 \%$ y $15 \%$, se mejora sustancialmente la durabilidad del concreto mediante el incremento en la rigidez del gel C-S-H. Estas observaciones concuerdan también con lo reportado cuando se por (Hosseni et. al., 2010), ellos trabajaron con morteros de ferrocementos a los que adicionaron NS en porcentajes de 1 a $3 \%$ en peso con respecto al cemento portland, obteniendo como resultado una mejora en la $\mathrm{RC}$ y una mayor densidad en la zona interfacial de transición, todo esto a relaciones a/c de 0.35 y 0.40 . Otros de los avances en los que se está incursionando con los nanomateriales, es su aplicación para mejorar el comportamiento de los agregados reciclados (Hosseni et. al., 2009). Al concluir su trabajo experimental reportó que si bien el uso de nanopartículas ayudaba a la reacción de hidratación, el uso de los agregados reciclados generaba resistencias y trabajabilidades menores a las obtenidas con un agregado nuevo cuando se dosificaban las nanopartículas de sílice en porcentajes de $1.5 \%$ y $3.0 \%$. Por otro lado, se estudió la formación de una película delgada las nanopartículas directamente sobre la superficie de los agregados (San Filippo et. al., 2009), obteniendo una mejora en la adherencia de los agregados y la pasta de cemento, teniendo como resultado, una mejora en general en las propiedades mecánicas. Adicionalmente reportan mejoras en las RC de hasta $35 \%$ con una adición de $0.032 \% \mathrm{SiO}_{2}$ depositado sobre la superficie de los agregados.

Como se ha mencionado anteriormente, el uso de la nanotecnología en la industria de la construcción permite modificar las propiedades del cemento y/o concreto elaborado con estos materiales. Sin embargo, un punto en común de la mayoría de los estudios lo constituye el proceso de adición de las nanopartículas y su correcta dispersión en la matriz. Como lo menciona (Sobolev et. al., 2009), la distribución de la nano- $\mathrm{SiO}_{2}$ en la pasta de cemento, juega un papel esencial y gobierna de manera total el desempeño de los productos obtenidos. En otro tipo de nanomateriales, como los nanotubos de carbono, el problema de la dispersión también está presente, (Shah et. al., 2009) detallaron que el mayor inconveniente de la incorporación de nanotubos de carbono a matrices cementantes es la pobre dispersión que se obtiene.

Es importante hacer mención que a través del presente artículo se presenta una contribución novedosa referente al estudio de un cemento sulfoaluminoso con adición de NS, ya que existe muy poca información relacionada con el tema (Raki et. al., 2010, Jewell, 2015 y Chung et. al., 2012). 
Revista ALCONPAT, Volumen 6, Número 2, Mayo - Agosto 2016, Páginas 101 - 115

Adicionalmente, el ataque químico por sulfatos en los dos tipos de cemento en presencia de NS es un tópico interesante que tampoco se ha estudiado ampliamente.

\section{PROCEDIMIENTO}

Se utilizaron dos matrices cementantes: (a) Cemento Portland Ordinario, CPO 40, de acuerdo a la clasificación de la Norma NMX-C-414-ONNCCE-2010 y, (b) Cemento sulfoaluminoso (CSA) cuya característica principal es que contiene, además de las fases tradicionales del cemento $\left(\mathrm{C}_{3} \mathrm{~S}, \mathrm{C}_{2} \mathrm{~S}, \mathrm{C}_{3} \mathrm{~A}\right.$ y $\left.\mathrm{C}_{4} \mathrm{AF}\right)$, sulfoaluminato de calcio $\left(\mathrm{C}_{4} \mathrm{~A}_{3} \mathrm{~S}\right)$. En la Tabla 1 , se presentan los resultados de la composición química de ambos cementos.

Tabla 1. Composición química de cementantes puros.

\begin{tabular}{|c|c|c|c|c|c|c|c|c|c|c|c|c|c|c|c|}
\hline Óxidos (\%p/p) & 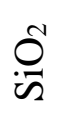 & $\frac{\bigcap_{\pi}}{\mathbb{Z}}$ & 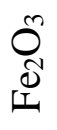 & 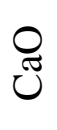 & $\sum_{\substack{0 \\
\sum}}^{0}$ & $\stackrel{\infty}{\infty}$ & 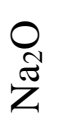 & $\begin{array}{l}\underset{\mathscr{N}}{0} \\
\underline{1}\end{array}$ & $\underset{\wp}{\mathscr{E}}$ & 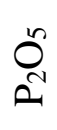 & 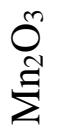 & 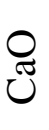 & $\vec{a}$ & نَ & $\stackrel{\oplus}{*}$ \\
\hline CPO 40 & 우 & $\hat{\sigma}$ & $\stackrel{\infty}{\stackrel{-}{-}}$ & \begin{tabular}{l}
\multirow{2}{*}{} \\
กี
\end{tabular} & $\stackrel{n}{n}$ & $\underset{\forall}{ \pm}$ & ?ొ? & $\begin{array}{l}\infty \\
0 \\
0\end{array}$ & तె & $\frac{m}{0}$ & $\begin{array}{l}\hat{8} \\
\stackrel{0}{0}\end{array}$ & $\stackrel{\Upsilon}{\Perp}$ & $\nabla$ & نج & $\begin{array}{l}m \\
\infty \\
\dot{0}\end{array}$ \\
\hline CSA & $\begin{array}{l}\hat{\sigma} \\
\infty \\
\infty\end{array}$ & $\underset{\forall}{\stackrel{\nabla}{*}}$ & بْ & $\frac{ \pm}{\dot{\sigma}}$ & $\begin{array}{l}m \\
\infty \\
0\end{array}$ & $\begin{array}{l}\bar{a} \\
\dot{n}\end{array}$ & $\stackrel{\Upsilon}{0}$ & $\frac{n}{0}$ & $\frac{\infty}{0}$ & 웅 & $\stackrel{\text { Oִ }}{0}$ & $\begin{array}{l}0 \\
0\end{array}$ & $\stackrel{\text { fே }}{6}$ & $\dddot{n}$ & 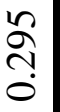 \\
\hline
\end{tabular}

* P.P.I.: pérdida de peso por ignición; R.I: Residuos insolubles; A.T.: álcalis totales.

De la Tabla 1 se puede observar que el contenido de $\mathrm{SO}_{3}$ de ambos cementos está en el rango del 4.0\%, siendo de $4.14 \%$ para el CPO y de $3.91 \%$ para el CSA. Se observa también que el contenido de $\mathrm{CaO}$ es ligeramente mayor en el cemento CSA que en el cemento CPO (63.14\% vs. $62.54 \%)$ lo que indica un mayor contenido de adición de caliza en el cemento CSA. La cuantificación de fases para ambos cementos se llevó a cabo mediante el método de refinamiento Rietveld, empleando para ello el Software HighScore Plus versión 3.05. Los resultados del refinamiento Rietveld se presentan en la Tabla 2, y de manera gráfica en la Figura 1. El resultado de la cuantificación mineralógica por Rietveld confirmó que los cementos CPO y CSA, presentan contenidos diferentes de los principales minerales del clinker, $\mathrm{C}_{3} \mathrm{~S}, \mathrm{C}_{2} \mathrm{~S}, \mathrm{C}_{3} \mathrm{~A}$. El análisis también mostró la presencia de $\mathrm{CaCO}_{3}$ como adición en ambos cementos aunque en el cemento CSA, fue posible determinarlo en contenidos de hasta $15 \%$ a diferencia del 2.5\% determinado para el cemento CPO. La principal diferencia mineralógica de los cementos lo constituye la Yelimita o $\mathrm{C}_{4} \mathrm{~A}_{3} \mathrm{~S}$, identificada únicamente en el cemento CSA en contenido de $1.5 \%$. Además se utilizó un aditivo de nanosílice comercial, cuya caracterización se presenta en la Tabla 3. 
Revista ALCONPAT, Volumen 6, Número 2, Mayo - Agosto 2016, Páginas 101 - 115

Tabla 2. Resultados de cuantificación Rietveld para los cementos de estudio.

\begin{tabular}{|l|c|c|c|}
\hline \multicolumn{1}{|c|}{ Componente } & Fórmula & CPO 40 & CSA \\
\hline $\mathrm{C}_{3} \mathrm{~S}$ - Alita de la Torre et al & $\mathrm{Ca}_{3} \mathrm{SiO}_{5}$ & 61.9 & 36.4 \\
\hline $\mathrm{C}_{2} \mathrm{~S}$ - Belita & $\mathrm{Ca}_{2} \mathrm{SiO}_{4}$ & 18.9 & 27.3 \\
\hline $\mathrm{C}_{3} \mathrm{~A}$ - Aluminato Cúbico & $\mathrm{Ca}_{9}\left(\mathrm{Al}_{6} \mathrm{O}_{18}\right)$ & 5.0 & 1.2 \\
\hline $\mathrm{C}_{3} \mathrm{~A}-$ Aluminato & $\mathrm{Ca}_{9}\left(\mathrm{Al}_{6} \mathrm{O}_{18}\right)$ & ---- & 1.8 \\
\hline Ferroaluminato de Calcio & $\mathrm{Ca}_{2} \mathrm{Fe}_{1.52} \mathrm{Al}_{0.48} \mathrm{O}_{5}$ & 3.7 & 3.7 \\
\hline Yelimita & $\mathrm{Ca}_{3} \mathrm{Al}_{6} \mathrm{O}_{2} \mathrm{CaSO}_{4}$ & ---- & 1.5 \\
\hline Anhidrita & $\mathrm{CaSO}_{4}$ & 0.8 & 3.2 \\
\hline Yeso & $\mathrm{CaSO}_{4} \cdot 2 \mathrm{H}_{2} \mathrm{O}$ & 7.2 & 5.5 \\
\hline Carbonato de Calcio & $\mathrm{CaCO}_{3}$ & 2.5 & 15.1 \\
\hline Óxido de Calcio & $\mathrm{CaO}$ & ---- & 4.3 \\
\hline \multicolumn{2}{|c|}{$\mathrm{GOF}$} & 8.6 & 11.2 \\
\hline
\end{tabular}

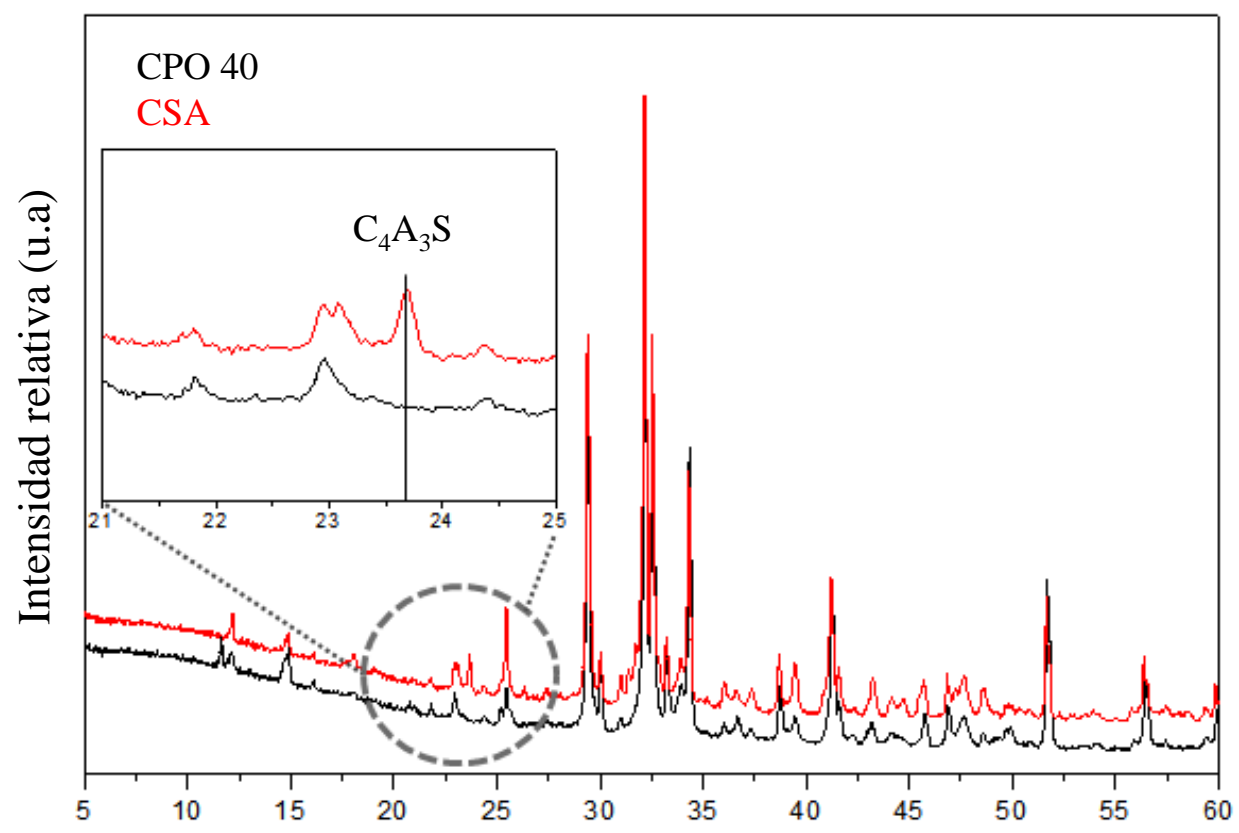

Posición angular $2 \theta$

Figura 1. Comparación patrones de difracción de cementos. En el detalle la fase $\mathrm{C}_{4} \mathrm{~A}_{3} \mathrm{~S}$ presente en el cemento

Las concentraciones de nanopartículas a utilizar fueron de $0 \%, 0.3 \%, 1 \%$ y $5 \%$ en peso con respecto al cemento. Cabe mencionar que aun cuando para este tipo de materiales, utilizar dosificaciones superiores al $1.0 \%$ resultarían por el momento poco viables debido al costo de estos materiales, en la presente investigación se utilizaron dichos niveles para determinar su influencia en las propiedades de los morteros. El proceso de mezclado utilizado fue similar al indicado en la Norma Norma NMX-C085-ONNCCE-2010, con una ligera modificación; se agregó el agua de mezclado, se adicionó el superplastificante (SP) y se mezcló por 60 s. Posteriormente se agregó la NS y se mezcló por $60 \mathrm{~s}$ y se continuó con el proceso como lo indica la norma: se agregó el cemento y al final la arena. Fue necesario emplear $0.5 \%$ de SP base policarboxilato con respecto al peso del cemento, para asegurar la dispersión de las NS. Después de $24 \mathrm{~h}$, las probetas se desmoldaron y se colocaron en una solución saturada de hidróxido de calcio (con la finalidad de evitar su lixiviación) para el proceso de curado. 
Revista ALCONPAT, Volumen 6, Número 2, Mayo - Agosto 2016, Páginas 101 - 115

Las muestras se caracterizaron mediante resistencia a la compresión (RC); posteriormente fracciones sólidas de las muestras se sumergieron en acetona y se secaron en vacío a $50^{\circ} \mathrm{C}$ durante $24 \mathrm{~h}$, con el fin de detener las reacciones de hidratación y analizarlas mediante microscopía electrónica de barrido (MEB). Las muestras fueron además caracterizadas mediante calorimetría isotérmica por conducción.

Tabla 3. Caracterización de aditivo líquido de nanosílice

\begin{tabular}{|l|c|c|}
\hline \multicolumn{1}{|c|}{ Característica } & Especificación & Resultado \\
\hline Forma & Líquido & Líquido \\
\hline Color & $\begin{array}{c}\text { Transparente } \\
\text { lechoso }\end{array}$ & $\begin{array}{c}\text { Transparente } \\
\text { lechoso }\end{array}$ \\
\hline Gravedad específica, $20^{\circ} \mathrm{C}$ & $1.134 \pm 0.03$ & 1.22 \\
\hline Viscosidad, $20^{\circ} \mathrm{C}, \mathrm{Brookfield}, \mathrm{Sp} 00 / 100 \mathrm{rpm}$ & $<30 \mathrm{cps}$ & $\mathrm{ND}$ \\
\hline $\mathrm{pH}$ & $10 \pm 1$ & 10.3 \\
\hline$\%$ Sólidos & ------------ & 32.5 \\
\hline
\end{tabular}

*Donde $N D=$ No determinado

\section{RESULTADOS}

\subsection{Calorimetría isotérmica}

Las pruebas de calorimetría isotérmica se realizaron a las pastas elaboradas con el cemento CPO 40 y con el cemento CSA. En la Tabla 4 se presentan las mezclas preparadas para la realización de la prueba de calorimetría isotérmica. A los cementos se les adicionó el SP en dosis de $0.50 \%$ y las nanopartículas en dosis de $1.0 \%$ y $5.0 \%$, con la finalidad de evaluar el efecto sobre el calor generado durante las reacciones de hidratación. Las pastas se realizaron con una relación agua/cemento $(\mathrm{a} / \mathrm{c})$ de 0.40 y 0.50 (ver Tabla 4).

Tabla 4. Muestras preparadas para las pruebas de calorimetría isotérmica de barrido por conducción cementos con NS y SP.

\begin{tabular}{|c|c|c|c|c|c|c|c|c|}
\hline Muestra & 1 & 2 & 3 & 4 & 5 & 6 & 7 & 8 \\
\hline Tipo de cemento & 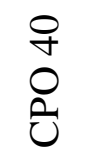 & $\begin{array}{l}\text { \& } \\
0 \\
0 \\
0\end{array}$ & $\underset{\mathscr{U}}{\mathbb{U}}$ & $\underset{\mho}{\mathbb{U}}$ & $\begin{array}{l}\text { ̊ } \\
0 \\
0\end{array}$ & $\begin{array}{l}\text { ̊ } \\
0 \\
0\end{array}$ & $\underset{\widetilde{U}}{\mathbb{U}}$ & $\underset{\widetilde{U}}{\mathscr{U}}$ \\
\hline $\mathrm{NS}(\% \mathrm{p} / \mathrm{p})$ & --- & --- & --- & --- & 1 & 5 & 1 & 5 \\
\hline $\mathrm{SP}(\% \mathrm{p} / \mathrm{p})$ & 0.5 & 0.5 & 0.5 & 0.5 & 0.5 & 0.5 & 0.5 & 0.5 \\
\hline $\mathrm{rel} \mathrm{a/c}$ & 0.5 & 0.4 & 0.5 & 0.4 & 0.5 & 0.4 & 0.5 & 0.4 \\
\hline
\end{tabular}


Revista ALCONPAT, Volumen 6, Número 2, Mayo - Agosto 2016, Páginas 101 - 115
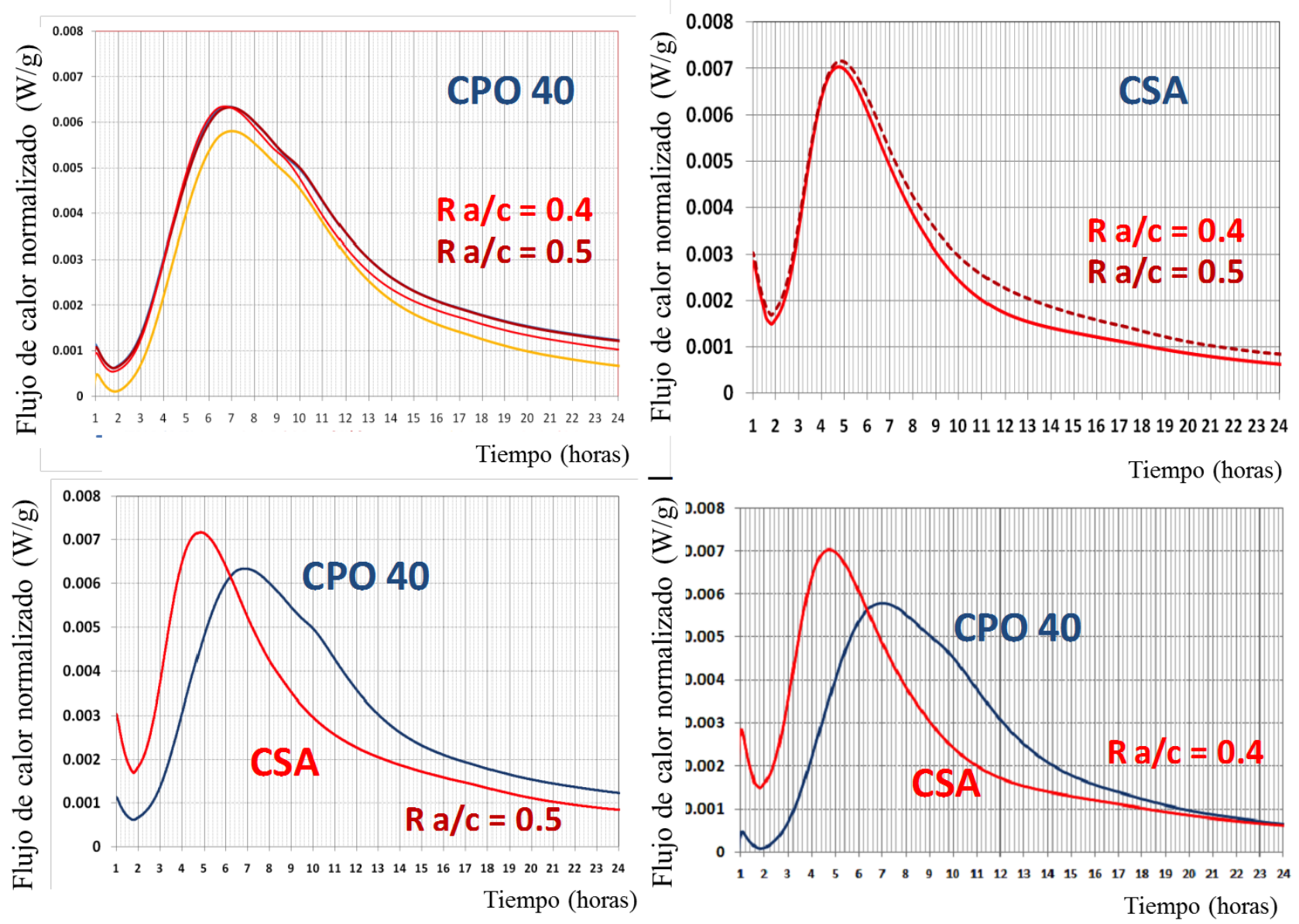

Figura 2. Calorimetría de los cementos a diferentes relaciones a/c.

En la Figura 2 se observa que el periodo de inducción terminó alrededor de las 2.0 horas para el cemento CPO 40 mientras que para el cemento CSA, terminó antes. Es en este tiempo cuando inicia el periodo de fraguado, la capa de C-S-H se rompe y el proceso de hidratación continúa. En los resultados obtenidos se observó que relaciones menores de a/c aceleran la reacción de hidratación ligeramente; este efecto es más pronunciado para el cemento CPO 40 que para el cemento CSA.

Como se puede observar en la Figura 2, el efecto que se presentó en ambos cementos al dosificar el $0.5 \%$ de SP, fue un desplazamiento de la curva de desprendimiento de calor, indicando un alargamiento del periodo durmiente, provocando que la curva se hiciera más pronunciada y con una menor cresta registrando el nivel máximo de desprendimiento de calor a las $15 \mathrm{~h}$ para el cemento CPO 40 con $0.5 \%$ de SP comparado con las $7 \mathrm{~h}$ que le toma al cemento CPO 40 sin SP alcanzar su punto más alto, es decir, la adición del $0.5 \%$ SP implica que tomará el doble de tiempo en iniciar con el desarrollo de RC. Un comportamiento similar es el mostrado para el cemento CSA; en donde el efecto del SP pareciera ser incluso un poco mayor, ya que el cemento sin SP registra su máximo desprendimiento de calor a las $4.7 \mathrm{~h}$, mientras que con la adición del $0.5 \% \mathrm{SP}$, el punto de máximo desprendimiento de calor se registra a las $13 \mathrm{~h}$, es decir, casi 3 veces más lento que el cemento de referencia. 
Revista ALCONPAT, Volumen 6, Número 2, Mayo - Agosto 2016, Páginas 101 - 115

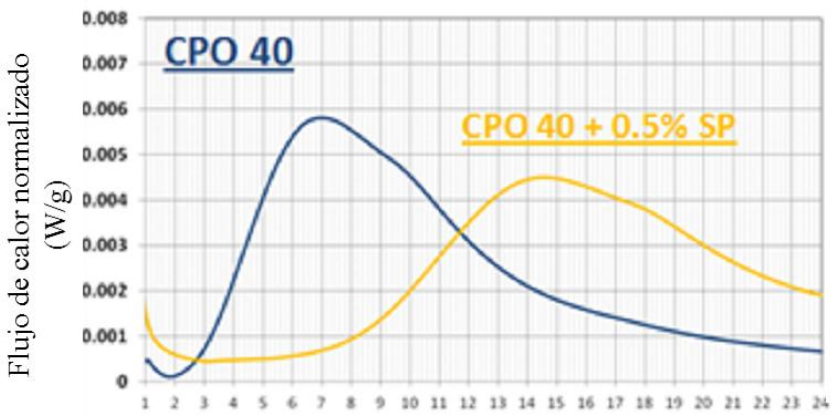

Tiempo (horas)

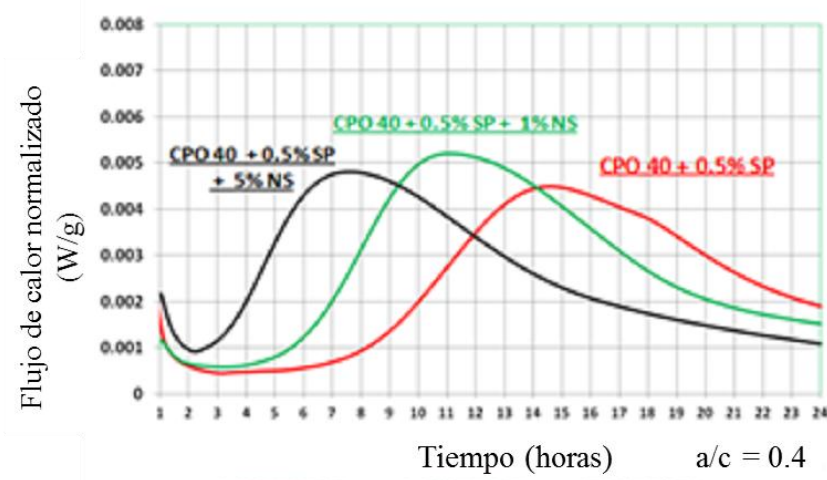

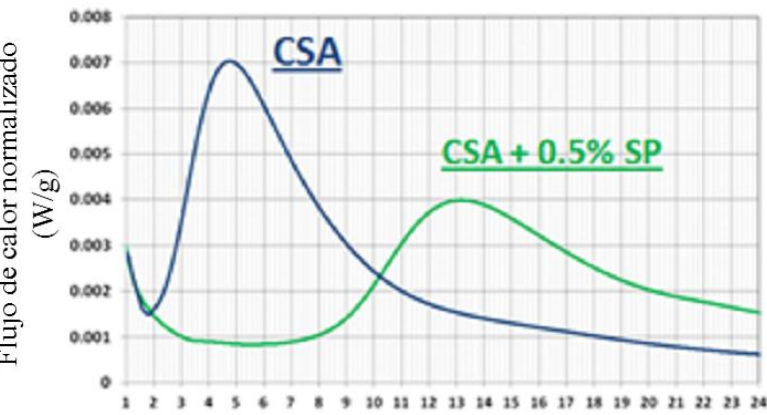

Tiempo (horas) $\quad \mathrm{a} / \mathrm{c}=0.4$

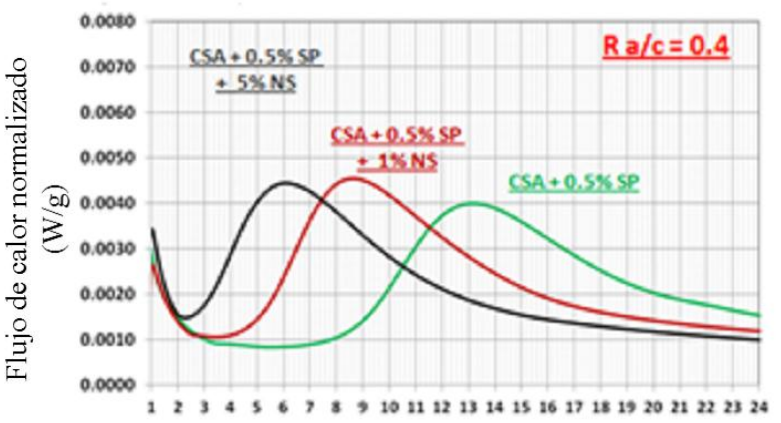

Tiempo (horas) $\quad \mathrm{a} / \mathrm{c}=0.4$

Figura 3. Calorimetría de los cementos adicionados con NS y SP; a/c de 0.40 .

Por otro lado, se observa que comparando los perfiles de calor de ambos cementos, con la misma relación a/c, el cemento CSA presenta una curva de evolución de calor muy diferente a la presentada por el cemento $\mathrm{CPO} 40$, presentando el pico principal debido a la reacción exotérmica del $\mathrm{C}_{3} \mathrm{~S}$ a las 6.5 $\mathrm{h}$ para el cemento CPO 40 y de $4.5 \mathrm{~h}$ para el cemento CSA. Adicionalmente, el pico exotérmico del cemento CSA alcanzó un flujo de calor máximo de $0.07 \mathrm{~W} / \mathrm{g}$ contra el $0.06 \mathrm{~W} / \mathrm{g}$ del CPO 40 . Esto indica que la reacción de hidratación del cemento CSA sucede con mayor rapidez y que la RC inicial debería ser mayor en el cemento CSA, sin embargo, los resultados obtenidos de RC a relación a/c constante mostraron una mayor RC a 24 horas para el cemento CPO 40 alcanzando $18.0 \mathrm{MPa}$ vs 13.1 $\mathrm{MPa}$ alcanzados por el cemento CSA. Esto puede explicarse por el efecto de dilución del cemento CSA, que de acuerdo a los resultados de la composición química, contiene un mayor contenido de caliza como adición, en comparación con el cemento CPO 40. Los resultados también muestran un tiempo de fraguado menor del CSA con respecto al obtenido con el cemento CPO 40. Una vez obtenidas las curvas calorimétricas de los cementos referencia sin la incorporación de aditivos y a las dos relaciones a/c, se determinó el calor desprendido por las mezclas de cemento con la incorporación de las NS.

Estos resultados confirman lo observado por Puertas (Puertas et. al., 2001 y Puertas et. al., 2005) en donde independientemente de la naturaleza del aditivo SP adicionado, todos ellos retrasaban las reacciones de hidratación de los silicatos de calcio, extendiendo los tiempos de fraguado. Este efecto de retraso puede ser explicado debido a un fenómeno de adsorción del polímero en los granos de cemento, formando una barrera o capa alrededor de dichos granos, impidiendo el paso de las moléculas de agua a las partículas de cemento, además de la formación de complejos entre los iones $\mathrm{Ca}^{+2}$ formados en la hidratación inicial y los aniones de los polímeros que afectan a la nucleación y precipitación del $\mathrm{Ca}(\mathrm{OH})_{2}$.

Sin embargo, la RC de las morteros con SP indicaron que a $24 \mathrm{~h}$, la RC del CPO 40 puro fue menor que la del que contenía $0.5 \% \mathrm{SP}$, con $18 \mathrm{MPa}$ y $23.5 \mathrm{MPa}$ respectivamente. 
Los resultados de calorimetría indican una extensión importante del tiempo de fraguado inicial, sin embargo, este resultado no necesariamente implicaría alguna relación con las RC esperadas por estas mezclas de cemento debido a que el proceso de fraguado y el proceso de desarrollo de $\mathrm{RC}$ son independientes uno del otro. Es importante mencionar que para el cemento CSA el efecto fue diferente, y la adición de SP disminuyó la RC a 24 horas, pasando de 13.1 a $8.2 \mathrm{MPa}$.

Se observa también en la Figura 3, que al adicionar las nanopartículas a una misma relación a/c, las curvas de evolución de calor se desplazan hacia menores tiempos, lo que sugeriría que los procesos de fraguado y de evolución de las reacciones de hidratación se ven acelerados por la adición de las nanopartículas de sílice. Esta tendencia se observó para ambos tipos de cemento.

Este comportamiento confirma lo reportado por (Qing et. al., 2007) cuando observaron que al incrementar el porcentaje de adición de partículas de nanosílice, la consistencia de la pasta decrecía, es decir, observaron que aceleró el proceso de hidratación en comparación con otros materiales puzolánicos como el humo de sílice. Otros autores (Björnström et. al., 2004, Morteza et. al., 2014 y Li et. al., 2004) encontraron que la sílice en tamaños nanométricos acelera el proceso de hidratación y la formación de gel C-S-H gracias a su elevada energía superficial; además, con el porcentaje de adición de la NS, se incrementa el calor de hidratación de la mezcla como consecuencia de la finura de las NS. De este modo, es posible observar también que a mayor contenido de nanopartículas adicionadas, es menor el tiempo en el que se alcanza el máximo desprendimiento de calor. De acuerdo a los datos del cemento CPO 40, el pasar de 0 a $1.0 \%$ NS, el tiempo en el que se alcanzó el nivel máximo de calor liberado bajó de $14.5 \mathrm{~h}$ a $11 \mathrm{~h}$ y al agregar NS en un 5\% el tiempo disminuyó de $11 \mathrm{~h}$ a $7.5 \mathrm{~h}$. Para el caso del CSA, el comportamiento, fue similar, de manera que sin adiciones el tiempo fue de $13.3 \mathrm{~h}$ con NS en $1 \%$ de $8.8 \mathrm{~h}$ y con $5 \%$ NS de $6 \mathrm{~h}$.

Este comportamiento observado experimentalmente tiene una forma parabólica y puede ser explicado a través de la siguiente ecuación:

$$
\mathbf{f}(\mathbf{x})=\mathbf{a x} \mathbf{x}^{2}+\mathbf{b x}+\mathbf{c}
$$

dónde : $\mathrm{f}(\mathrm{x})$ = tiempo de máxima liberación de calor, $\mathrm{x}=\%$ de nanopartículas; $\mathrm{a}$, b y c son coeficientes de cada uno de los sistemas.

Ecuación para el sistema con CPO 40:

$$
\text { TTMáx }=0.525(\% N S)^{2}-4.025(\% N S)+14.5
$$

Ecuación para el sistema con CSA:

$$
\text { TTMáx }=0.76(\% N S)^{2}-5.26(\% N S)+13.3
$$

De la Figura 4 se puede concluir que, si bien al agregar las nanopartículas a la matriz cementante acelera las reacciones de hidratación y por ende es de esperarse un incremento en la RC, llega un punto en que aun cuando se continúe incrementando el contenido de nanopartículas, no se tendrá el efecto de aceleración de la reacción. En la Figura 4 también puede observarse que en dosificaciones mayores a $3.5 \%$ de NS, la velocidad a la que se alcanza el máximo desprendimiento de calor comienza a incrementarse nuevamente. Esto confirmaría que las nanopartículas aceleran el desarrollo de las RC a las edades iniciales siempre y cuando no se exceda este porcentaje. 
Revista ALCONPAT, Volumen 6, Número 2, Mayo - Agosto 2016, Páginas 101 - 115

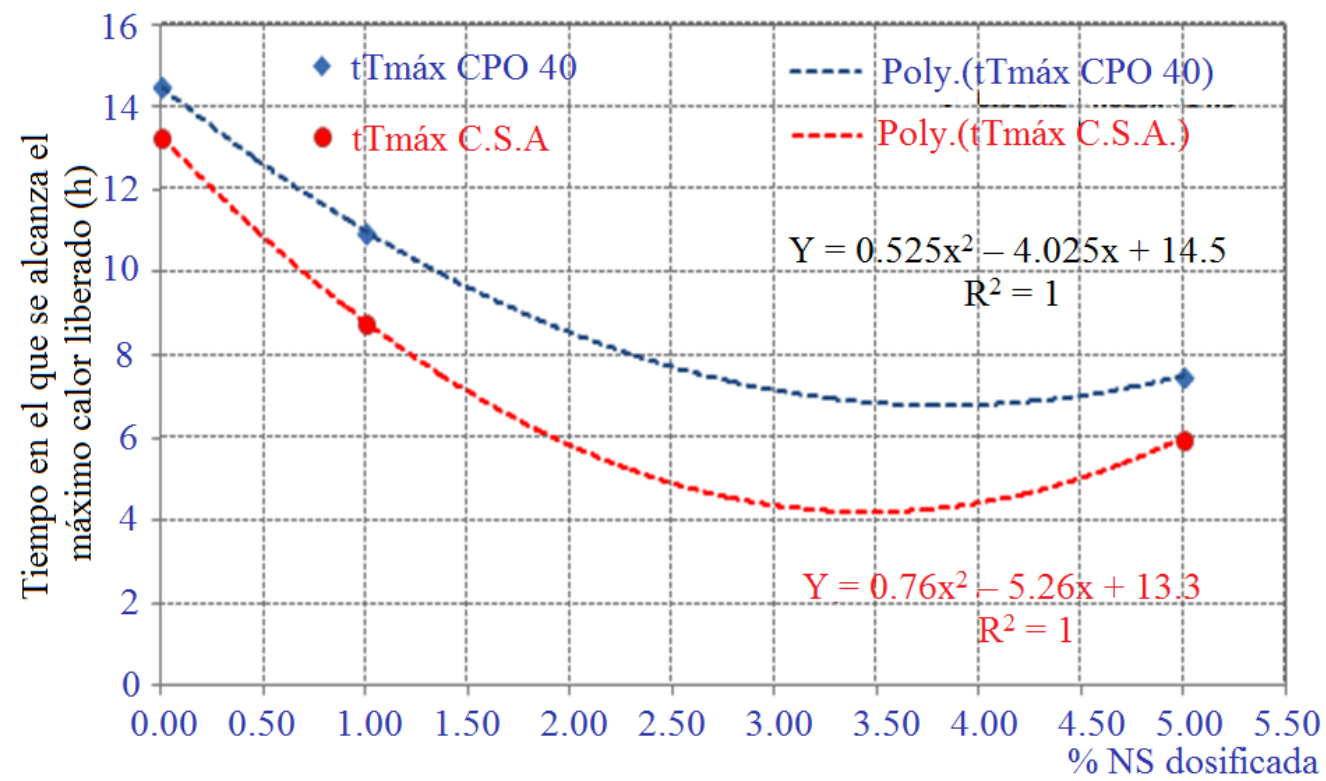

Figura 4. Comportamiento de tiempo de máxima liberación de calor en función de la adición de NS.

\subsection{Resistencias a la compresión}

Para realizar los ensayos de RC se elaboraron muestras con dosificaciones de $0.5 \%$ de SP y adiciones de nanopartículas de $0.3 \%, 1 \%$ y $5 \%$. La dosificación de $5 \%$ se eligió con la finalidad de provocar una modificación importante en las propiedades mecánicas del cemento elaborado. Los resultados de la RC a 24 h, 3, 7, 28, 90 y 150 días, se presentan en la Figura 5. Es importante señalar que para esta primera serie de resultados, el agua adicionada a la mezcla se corrigió considerando el agua aportada por las NS que se encuentra originalmente en una suspensión, con la finalidad de mantener la relación a/c constante en 0.485 .

De los resultados se puede observar que, para el cemento CPO 40, la mejor RC a todas las edades se obtuvieron de la mezcla de cemento con el SP únicamente. De las mezclas que contenían adición de NS, se observa que a la edad de $24 \mathrm{~h}$, todas las mezclas preparadas superan a los resultados obtenidos por la referencia, mejorando su valor en hasta en un $43 \%$ (25.8 $\mathrm{MPa}$ vs $18 \mathrm{MPa}$ ) para la muestra con $5 \%$ de NS. A partir de la edad de 3 días, estos porcentajes disminuyen, teniendo un $15 \%$ de mejora para las mezclas 5\% de NS (40 MPa vs $34.9 \mathrm{MPa}$ de la referencia). A 7 días, se alcanzó un $11 \%$ de mejora en la RC para las muestra con SP mientras que la muestra con 5\% de NS desarrolló un $8 \%$ más de RC que la obtenida por la muestra de referencia. A 28 días, las muestras con SP desarrollaron un $17 \%$ más de RC que la referencia (54.9 vs $47.1 \mathrm{MPa})$, mientras que el incremento para las muestras con NS fue del orden de $11 \%$ con respecto a la referencia (52.1 y $52.3 \mathrm{MPa}$ para las muestras con $0.3 \%$ de NS y 5\% de NS contra 47.1 MPa de la referencia). Después de 150 días de curado, todas las muestras con NS alcanzan prácticamente el mismo valor que el obtenido por la referencia y únicamente la muestra con la adición de SP desarrolla un $18 \%$ de RC a la compresión mayor que la referencia. 

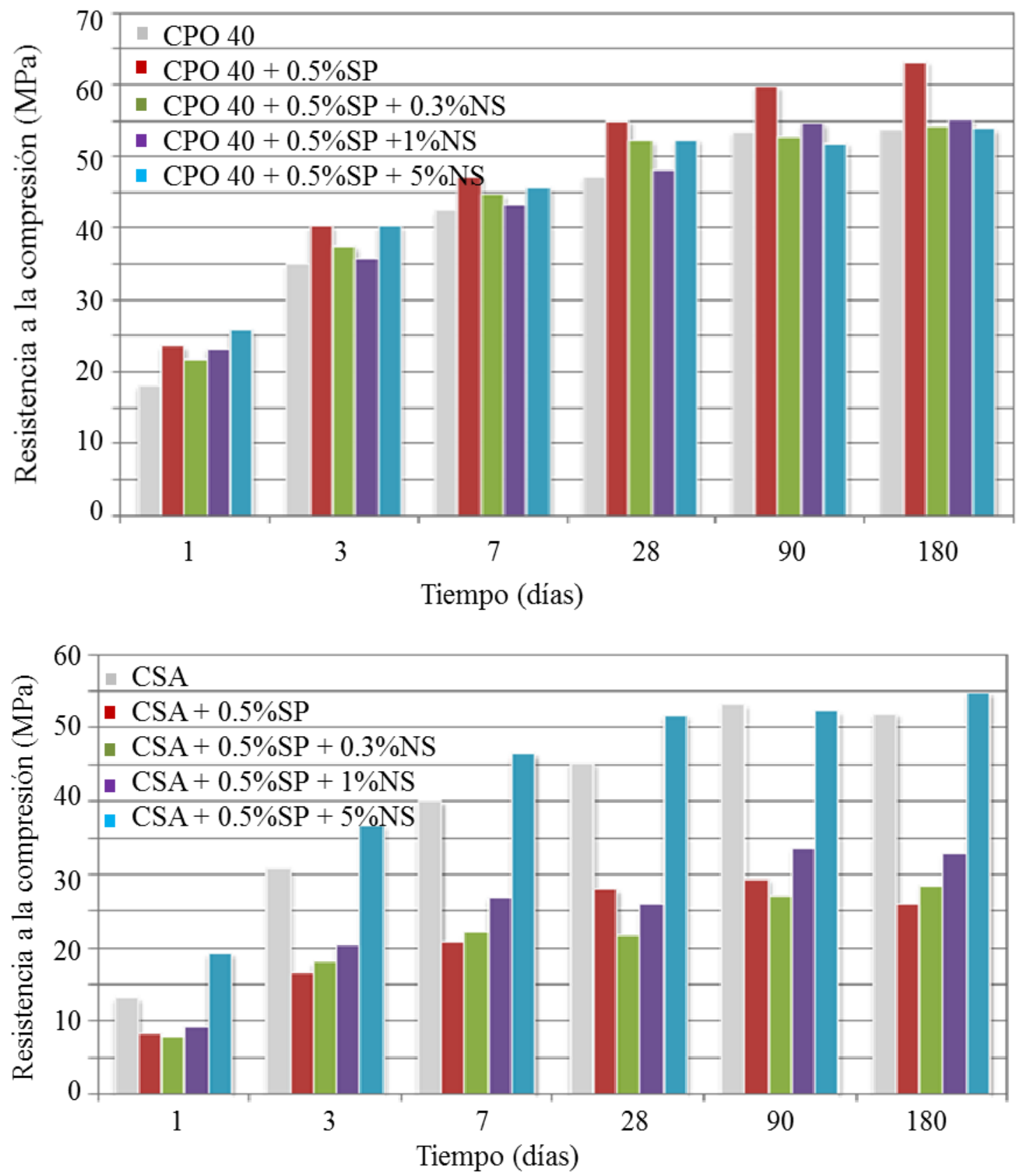

Figura 5. Resultados de resistencias a la compresión de los cementos CPO 40 y CSA sin ajuste de la cantidad de agua.

El efecto mostrado para la matriz de cemento CSA, fue diferente al presentado por el cemento CPO 40, debido a que la adición del SP, no favorecía el desarrollo de la RC, sino más bien inhibió los procesos de hidratación para esta matriz. Esto se puede observar en todos los resultados de las mezclas con $0.5 \%$ de SP, donde el desarrollo de RC con respecto a la referencia fue del orden de $63 \%, 54 \%$, $52 \%, 62 \%$ y $55 \%$ a las edades de 24 h, 3, 7, 28, 90 y 150 días respectivamente, debido a la disminución en la reactividad antes mencionada, ya que los SP están diseñados para cemento portland ordinario y las fases con sulfatos contenidas en el CSA pueden afectar de forma negativa la funcionalidad del SP. 
Revista ALCONPAT, Volumen 6, Número 2, Mayo - Agosto 2016, Páginas 101 - 115

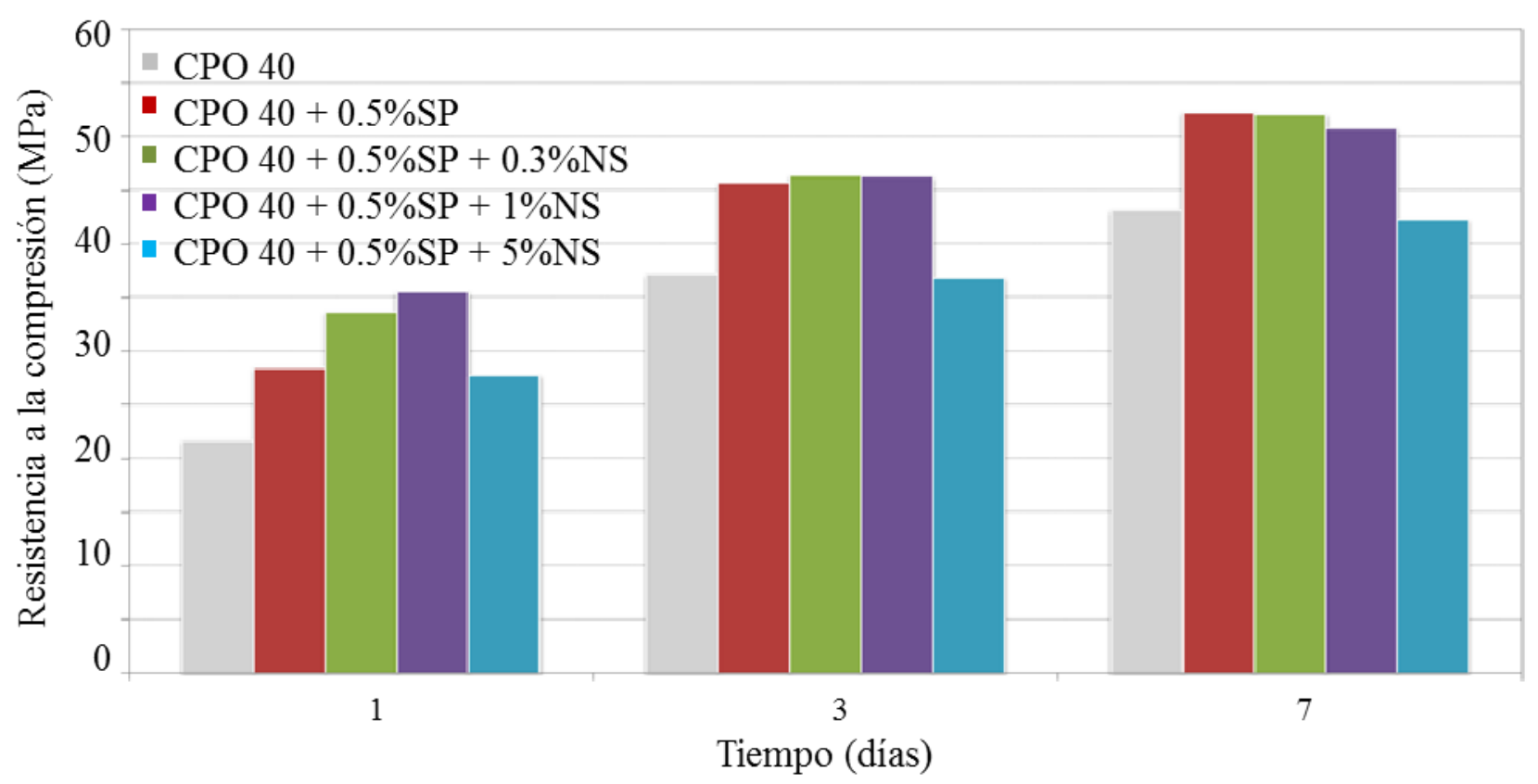

Figura 6. Resultados de resistencias a la compresión del cemento CPO 40 a fluidez constante.

Para esta matriz, se observó este comportamiento en todas las muestras con excepción de la mezcla con 5\% NS, en donde fue incluso posible observar un incremento en la RC del orden de $47 \%$ a $24 \mathrm{~h}$, $19 \%$ a 3 días, $16 \%$ a 7 días, 14\% a 28 días. De acuerdo con (Ma et. al., 2014) al adicionar SP en matrices de cemento belítico-sulfoaluminoso, la RC a 24 h disminuyó en todas las dosis que ensayaron, sin embargo, reportaron que a 28 días se obtenían RC equivalentes que las de la referencia e incluso superior cuando se dosificó en porcentaje de $0.075 \%$. Esto fue a que se retardó la formación de etringita a 1 día de hidratación, especialmente a concentraciones elevadas, obteniendo como resultado RC pobres. Esto podría explicar que la dosis de $0.5 \%$ para el cemento CSA utilizada en la presente investigación puede resultar alta para la naturaleza del cemento.

Como se puede observar en la Figura 6, a valores de fluidez similares, mediante el ajuste del agua de mezclado para obtener la misma fluidez; los resultados de RC del CPO 40 con la adición de NS cambian con respecto a lo observado anteriormente, si bien los mejores resultados se observan con la muestra de cemento con SP, los resultados con la adición NS son muy similares, e incluso resultan superiores a 24 horas para las adiciones de $0.3 \%$ y de $1 \%$ NS.

Estos resultados son relevantes porque nos plantea una observación sobre las especificaciones que marca la norma mexicana de cemento sobre el consumo de agua a utilizar cuando se trata de evaluar cementos ordinarios. Como se sabe, la norma indica que al tratarse de un cemento ordinario, la relación $\mathrm{a} / \mathrm{c}$ constante recomendada para evaluar las $\mathrm{RC}$ es de 0.485 . Los resultados obtenidos en la presente investigación sugieren que para sistemas con aditivos SP, o con sistemas que utilicen nanopartículas, lo más recomendable es buscar la fluidez y el agua necesaria para ajustar a esta fluidez y no utilizar una relación fija de a/c.

\subsection{Resistencia química al ataque por sulfatos}

La prueba para determinar la resistencia al ataque químico por sulfatos se realiza de acuerdo al procedimiento indicado en la Norma ASTM-C-1012.

En las figuras 7 y 8 , se presentan los resultados obtenidos para cada tipo de cemento en mediciones llevadas a cabo durante 550 días, cabe mencionar que la norma establece una duración mínima de 12 meses.Se puede observar que tanto el cemento CPO 40 como el cemento CSA no presentan una buena 
Revista ALCONPAT, Volumen 6, Número 2, Mayo - Agosto 2016, Páginas 101 - 115

resistencia al ataque químico por sulfatos, ya que el cambio en longitud excede el valor permitido por la norma NMX-C-414-ONNCE-2010 de expansión de $0.1 \%$ a 12 meses desde los 125 días para el cemento CPO 40 y desde los 50 días para el cemento CSA.

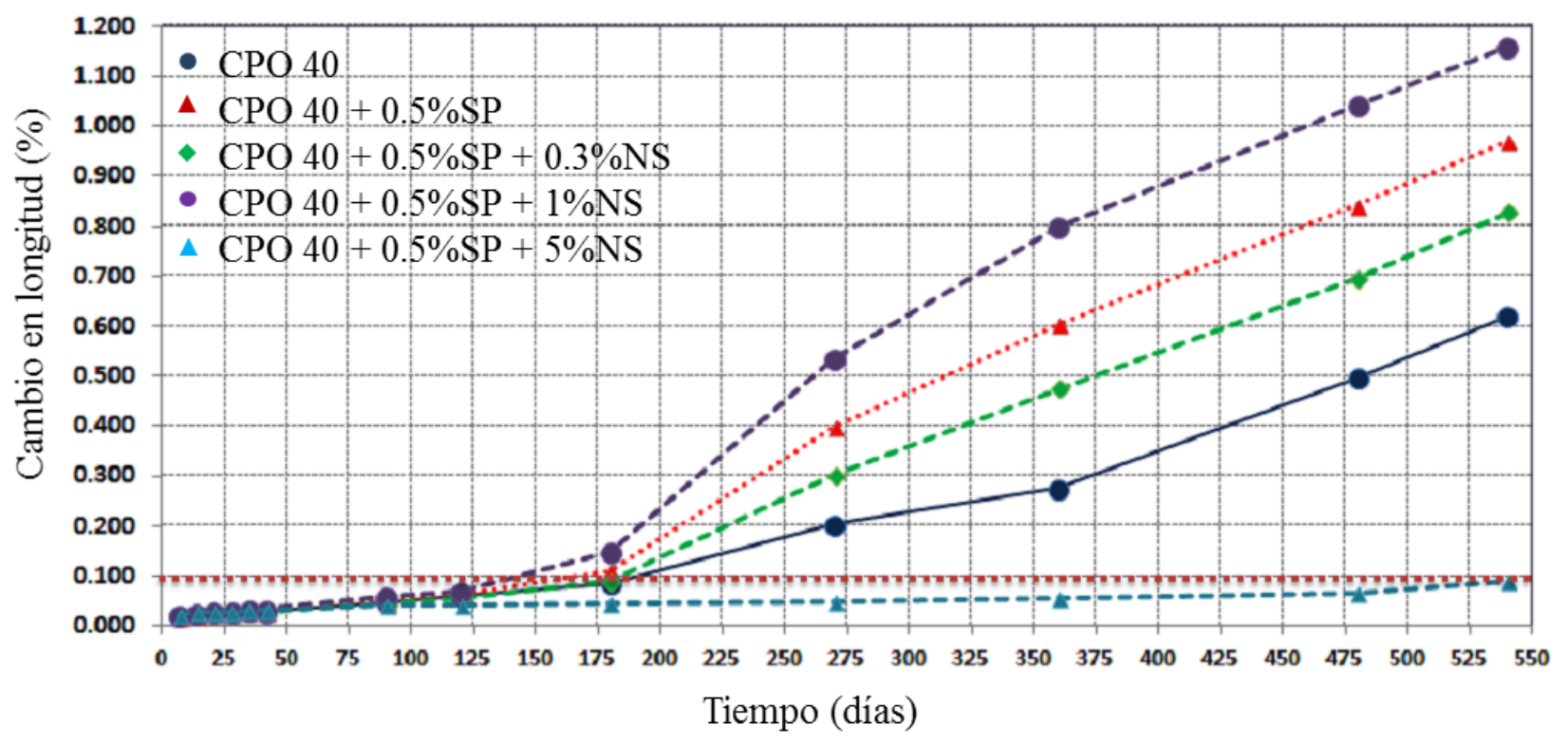

Figura 7. Resistencia al ataque químico por sulfatos, cemento CPO, 550 días de exposición.

El adicionar las nanopartículas en porcentajes de 5\%, favorece la resistencia al ataque químico por sulfatos, para ambos tipos de cemento. En el caso del cemento CPO 40, el beneficio de adicionar las nanopartículas es tan favorable que dicho cemento pasa de ser un cemento sin resistencia al ataque químico por sulfatos ("RS" en la nomenclatura utilizada en la norma mexicana NMX-C-414-2010ONNCCE a ser un cemento resistente a los sulfatos hasta al menos 550 días, con contenidos de $0.5 \%$ de SP y 5\% de NS. Para el caso del cemento CPO 40, la adición de nanopartículas en porcentajes menores al 5\%, no ofrece una mejoría en la resistencia al ataque químico.

Para el caso del cemento CSA, la adición de nanopartículas a la matriz cementante disminuyó la resistencia al ataque químico por sulfatos, ya que como se mencionó anteriormente, el cemento CSA sin adición de otros componentes, a partir de los dos meses excedía el límite establecido por la norma NMX-C-414-2010-ONNCCE. Sin embargo, al adicionar SP y NS, el cemento se mantuvo con características de resistencia al ataque químico hasta 275 días (9 Meses). Este cambio fue de un $94.8 \%$ para 180 días, que fue la máxima edad en la que el espécimen del cemento referencia mantuvo estabilidad volumétrica con respecto al CSA de referencia.

De acuerdo a los resultados se observó que para el CPO 40 la adición tanto de SP como de NS, ésta última en dosificaciones bajas, no resultó beneficiosa en términos de resistencia al ataque químico por sulfatos, en cambio, todas las adiciones en el CSA mejoraron su resistencia química comparándolo con el CSA puro. 
Revista ALCONPAT, Volumen 6, Número 2, Mayo - Agosto 2016, Páginas 101 - 115

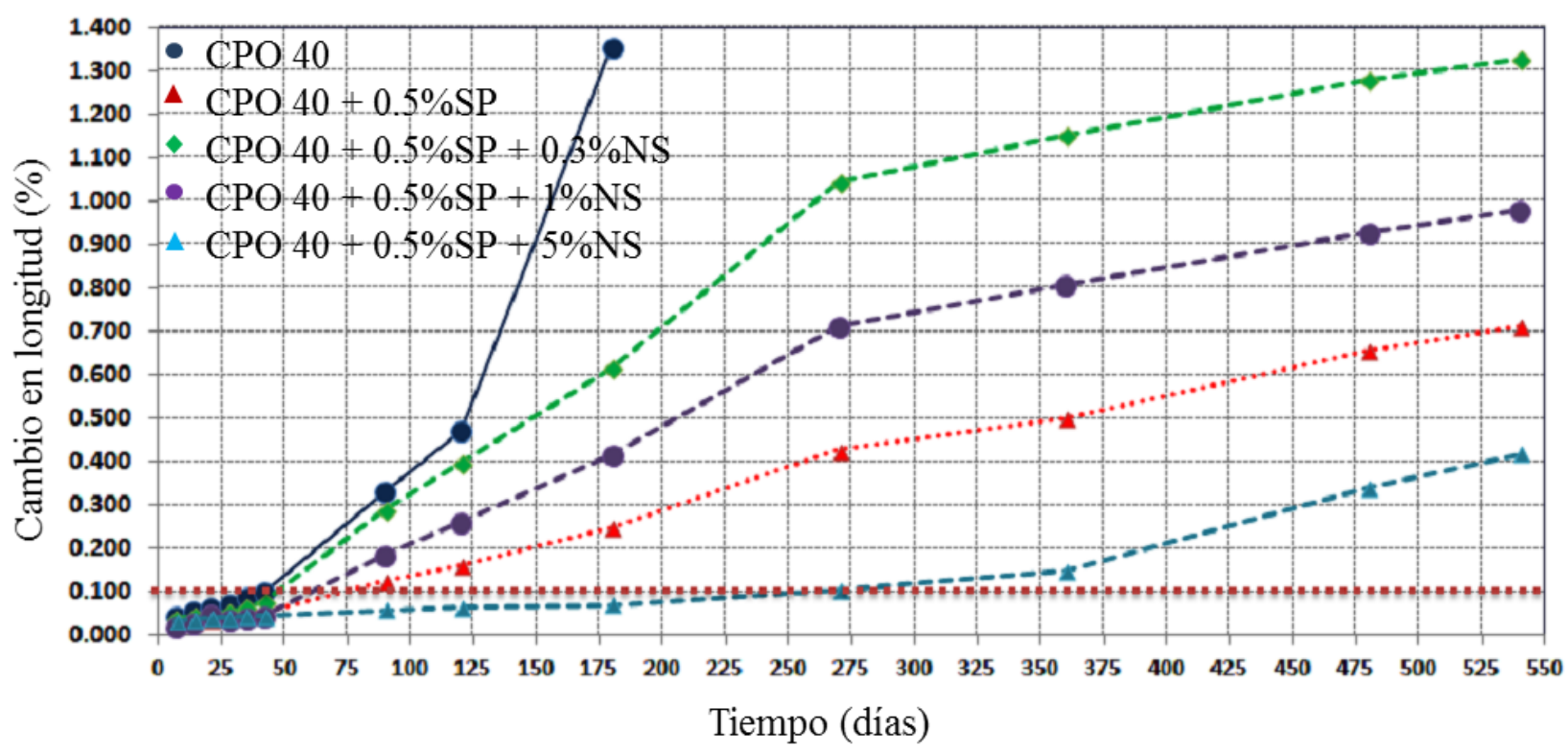

Figura 8. Resistencia al ataque químico por sulfatos, cemento CSA, 550 días de exposición.

Como se mencionó anteriormente, este comportamiento se podría explicar por una densificación de la matriz, la cual incrementó con el contenido de NS, y por ende se observó una reducción en la porosidad y la transferencia o el ataque de los iones sulfato tiende a ser más lento que en las muestras de cemento sin adición de nanopartículas. De nuevo, las nanopartículas estarían actuando como centros de nucleación para la generación de más gel C-S-H promoviendo esta disminución en la porosidad. Este comportamiento lo confirmaron otros autores (Quercia et. al., 2012 y Li et. al., 2004), reportando que la adición de nanopartículas incrementa la RC y disminuye de manera general la permeabilidad del concreto endurecido gracias a la reacción puzolánica, lo que resulta una mayor formación de C-S-H y en una estructura más densa. Utilizando montmorillonitas modificadas, se observó también que la permeabilidad se veía reducida hasta en 100 veces comparadas con los cementos tradicionales (Khater et. al., 2006),

De este modo, la mejora en la durabilidad de las muestras elaboradas con las NS se presenta como una característica que es posible modificar con el uso de estos materiales. La razón para esta mejora en la durabilidad, puede ser explicada debido a que las NS rellenan los poros del cemento, densificando la matriz y disminuyendo la porosidad y su permeabilidad, esto a su vez, incrementa la RC, tal como lo hace el humo de sílice.

\section{CONCLUSIONES}

- Las relaciones menores de a/c, aceleran la reacción de hidratación y este efecto fue más pronunciado para el CPO 40 que para el CSA.

- El CSA presenta una mayor velocidad de hidratación que el cemento CPO 40, y por consecuencia mayores RC iniciales.

- La adición de SP retrasa las reacciones de hidratación del cemento y este efecto se ve revertido a medida que se adiciona un mayor \%NS a las matrices cementantes.

- Las NS adicionadas a las matrices cementantes presentan un efecto acelerante sobre las reacciones de hidratación, sin embargo, este efecto puede revertirse a mayor contenido de NS. En los sistemas estudiados, el nivel de dosificación en donde se tiene un efecto de aceleración es hasta un 3.5\% de NS. 
Revista ALCONPAT, Volumen 6, Número 2, Mayo - Agosto 2016, Páginas 101 - 115

- Se observó que la adición de SP mejora sustancialmente la RC del CPO 40, para el caso del CSA, el comportamiento fue el contrario, con la adición de SP, el cemento desarrolló solamente el 50\% de la RC alcanzada por la referencia, excepto para la adición de $5 \% \mathrm{NS}$, en donde los resultados fueron superiores al CSA puro. Estos resultados se obtienen cuando la relación a/c se mantiene constante.

- Cuando se busca igualar la fluidez de los cementos, los resultados indican que con la adición de $1 \%$ NS en valores de $1.0 \%$, se mejora la RC hasta en alrededor de un $60 \%$ en comparación a los cementos sin adiciones; debido a su efecto de aceleración y su comportamiento puzolánico, el cual fue más pronunciado a edades iniciales, tal como lo reportado en los resultados de calorimetría.

- En general se observó que al agregar NS en ambas matrices, el ataque por sulfatos no fue tan pronunciado en comparación a las muestras sin adiciones.

- En el caso del cemento CPO 40 esta adición confiere al cemento la característica de resistencia al ataque químico por sulfatos, cuando originalmente es un cemento ordinario; lo que aumenta su valor agregado. Sin embargo, porcentajes menores al 5\%, no ofrecen ventaja en la resistencia al ataque químico por sulfatos para el cemento CPO 40.

- Para el caso del cemento CSA, la presencia de NS redujo el ataque químico por sulfatos, hasta en un $95 \%$. Esto es relevante, ya que el CSA sin la adición de algún material adicional, a los dos meses excede el límite establecido por la Norma Mexicana para un cemento resistente a los sulfatos.

\section{AGRADECIMIENTOS}

Se agradece ampliamente el soporte financiero de CONACYT y CEMEX para la realización de este proyecto.

\section{REFERENCIAS}

ASTM C1012/C1012M - 15, Standard Test Method for Length Change of Hydraulic-Cement Mortars Exposed to a Sulfate Solution.

Belkowit J. S., Armentrout D. (2010) "An investigation of Nano Silica in the Cement Hydration Process", Proceeding 2010 Concrete Sustainability Conference, National Ready Mixed Concrete Association, U.S.A., pp. $1-15$

Björnström J., Martinelli J., Matic A., Borjesson L., Panas I. (2004), “Accelerating effects of colloidal nanoSilica for beneficial calcium-silicate-hydrate formation in cement”, Chemistry Physic Letters; 392, pp. 242-248 Chung D. L. (2012) "Carbon materials for structural self-sensing, electromagnetic shielding and thermal interfacing”, Elsevier, CARBON 50, pp. 3342-3353

Hosseni P., Booshehrian A., Delkash M., Ghavami S., Zanjani M. K. (2009), "Use of Nano-SiO2 to Improve Microstructure and Compressive Strength of Recycled Aggregate Concretes", Nanotechnology in Construction 3, pp 215-221

Hosseni P., Booshehrian A., Farshchi S. (2010), "Influence of Nano-SiO2 addition on Microstructure and mechanical Properties of Cement Mortars for Ferrocement", Transportation Research Record; Journal of the transportation Research Board No. 2141, pp. 15-20

Jewell R. B. (2015) "Influence of Calcium Sulfoaluminate Cement on the Pullout Performance of Reinforcing Fibers: An Evaluation of the Micro-Mechanical Behavior", $\mathrm{PhD}$ Thesis, Civil Engineering, University of Kentuky.

Li H., Xiao H., Yuan J., Ou J. (2004) “Microstructure of cement mortar with nano-particles", Composites Part B: Engineering, 35, pp. 185-189

Ma B., Ma M., Shen X., Li X., Wu X. (2014), “Compatibility between a polycarboxylate superplasticizer and the belite-rich sulfoaluminate cement: Setting time and the hydration properties", Construction and Building Materials, 51, pp. 47-54 
Revista ALCONPAT, Volumen 6, Número 2, Mayo - Agosto 2016, Páginas 101 - 115

Mondal P., Shah S. P., Marks L. D., Gaitero J. J. (2010), "Comparative Study of the effect of Microsilica and Nanosilica in concrete", Transportation Research Record; Journal of the transportation Research Board No. 2141, pp. 6-9

Morteza B., Baghbadrani M., Aslani F. (2014), "Performance of nano-Silica modified high strength concrete at elevated temperatures", Construction and Building Materials, 68, pp. 402-408

Norma NMX-C-085-ONNCCE-2010, Industria de la construcción - Cementos hidráulicos - Determinación estándar para el mezclado de pastas y morteros de cementantes hidráulicos

Norma NMX-C-414-ONNCCE-2010, Industria de la construcción-Cementantes- Especificaciones y método de ensayo.

Puertas F., Vázquez T. (2001), "Hidratación inicial del cemento. Efecto de aditivos superplastificantes", Materiales de Construcción 51(262), pp 53-61.

Puertas F., Santos H., Palacios M., Martínez S. (2005), "Polycarboxylate superplaticizer admixtures: effect on hydration, microestructure and rheological behavior in cement pastes", Advances in Cement Research, 17, pp. 77-89

Qing Y., Zenan Z., Deyu K., Rongshen C. (2007), "Influence of nano-SiO ${ }_{2}$ addition on properties of hardened cement paste as compared with silica fume", Construction and Building Materials 21(3), pp. 539-545

Quercia G., Zpuesz P., Hüsken G., Brouwers J. (2012), "Effects of Amorphous Nano-Silica additions on Mechanical and Durability Performance of SCC Mixtures", Proceedings of the International Congress on Durability of Concrete (ICDC 2012), 18-21 June, Trondheim, Norway, pp. A2-A4

Raki L., Beaudoin J., Alizadeh R., Makar J. Sato T. (2010), "Cement and Concrete Nanoscience and Nanotechnology", Materials, 3(2), 918-942

San Filippo J. M., Muñoz J. F., Isabel Tejedor M., Anderson M. A., Cramer S. M. (2009), "Nanotechnology to Manipulate the aggregate-Cement Paste Bond Effects on Mortar Performance", Nanotechnology in Construction 3, pp. 29-33

Shah S. P., Konsta-Gdoutos M. S., Metaxa Z. S., Mondal P. (2009), "Nanoscale Modification of Cementitious Materials", Nanotechnology in Construction 3, Proceedings of the NICOM3, pp. 125-130

Sobolev K., Flores I., Torres-Martinez L. M., Valdez P. L., Zarazua E., Cuellar E. L. (2009) "Engineering of $\mathrm{SiO}_{2}$ Nanoparticles for Optimal Performance in Nano Cement-Based Materials"; Proceedings of the Nanotechnology in Construction 3 (NICOM3) 01/2009; pp. 139-148. 